\title{
Chemically addressed switching measurements in graphene electrode memristive devices using in situ XPS
}

\author{
Itır Köymen, (D) *a Pınar Aydoğan Göktürk, (DD b Coşkun Kocabaş ${ }^{c}$ \\ and Şefik Süzer iD ${ }^{b}$
}

Received 19th July 2018, Accepted 6th August 2018

DOI: $10.1039 / c 8 f d 00129 d$

The memristor has been the topic of heated research activity since the $\mathrm{Pt} / \mathrm{TiO}_{2} / \mathrm{TiO}_{x} / \mathrm{Pt}$ structure was reported by Hewlett-Packard Labs. The characteristics of memristors such as the pinched hysteresis loops and time and input signal-dependent memristance are due to the drift of positively charged oxygen vacancies in the $\mathrm{TiO}_{x}$ layer. While different modes of switching behaviour have been characterized, observing the switching as it happens with in situ measurements using X-ray photoelectron spectroscopy (XPS) can allow a better understanding of the device operation. The setup used in this work enables the application of voltage signals of different frequencies and amplitudes and observing the hysteresis seen in the $I-V$ plane through chemical addressing. The measurements were conducted on $\mathrm{Pt} / \mathrm{TiO}_{2} / \mathrm{TiO}_{x} /$ graphene structures. The single layer graphene, utilized as a top electrode, effectively acts as a transparent electrode in that the layer beneath it can be observed to a depth of $\sim 10 \mathrm{~nm}$ in XPS. This allows for the observation of the changes in the binding energies of $\mathrm{C} 1 \mathrm{~s}, \mathrm{Ti} 2 \mathrm{p}$ and $\mathrm{O} 1 \mathrm{~s}$. By comparing the variation in the binding energy of $\mathrm{Ti} 2 \mathrm{p}$ to that of $\mathrm{C} 1 \mathrm{~s}$, and observing how the variation changes for different excitation signals (at different frequency and amplitudes), it is possible to inspect the effect of the oxygen vacancy drift. We employed a variety of input signals with varying frequency and amplitudes in order to test the memristive devices thoroughly: sine wave, triangular wave and DC bias. Graphene has been attracting attention due to its intriguing optoelectronic properties. This study utilizes graphene as a transparent top electrode for in situ measurements in XPS to observe chemically-addressed memristive hysteresis while an excitation signal is being applied to the device.

\section{Introduction}

The memristor, theorised by Prof. Chua in 1971, has been recognised as the fourth fundamental circuit element, relating charge to flux. ${ }^{1}$ Since Hewlett

${ }^{a}$ Electrical and Electronics Engineering Department, Bilkent University, Ankara 06800, Turkey. E-mail: itirkoymen@ee.bilkent.edu.tr

${ }^{b}$ Chemistry Department, Bilkent University, Ankara 06800, Turkey

${ }^{\circ}$ School of Materials, University of Manchester, M13 9PL, UK 
Packard Labs reported the fabrication and characterisation of the first physical memristor, ${ }^{2}$ there has been a significant drive to understand, ${ }^{3}$ model $^{4,5}$ and utilise the device. ${ }^{6-8}$ The many attractive properties of memristors, such as nanometric dimensions, memory and CMOS compatibility, have rendered them a hot topic. Redox-based resistive switching random-access memories (ReRAMs) and phase change memories (PCMs) also exhibit memristive dynamics and switching, and are therefore recognised as being suitable for similar applications such as neuromorphic systems. ${ }^{9}$

Though memristive behaviour can be generalised and summarised with overarching characteristics (zero-crossing hysteresis loops on the first and third quadrants of the current-voltage plane), ${ }^{\mathbf{1 , 1 0}}$ and this type of behaviour has been observed with differing structures, ${ }^{2,11}$ a generalised model of a memristive device has still not been developed. Moreover, memristive behaviour which is not typical, i.e. non-zero-crossing hysteretic behaviour reminiscent of memristance, has been observed and has been recognised as the nanobattery effect. ${ }^{12}$ The lack of a generalised and flexible model means that circuit simulation software such as Cadence Spectre ${ }^{\circledR}$, SPICE etc. do not have defined models of the memristor. The derivation of such a model would facilitate and encourage the design of electronics employing memristors and memristive dynamics.

Memristors can be bistate (two resistance values: effectively $R_{\mathrm{ON}}$ (low resistance) and $R_{\mathrm{OFF}}$ (high resistance)), multi-state (several resistance values with boundary resistance which can be dubbed $R_{\mathrm{ON}}$ and $R_{\mathrm{OFF}}$ ) or analogue devices (continuous resistance change bound by $R_{\mathrm{ON}}$ and $R_{\mathrm{OFF}}$ ). If the switching mechanisms in these devices are well understood, the manufacturability of more predictable and reliable devices will be possible. Switching mechanisms and valence change in memristive structures and ReRAMs have been and are being studied using a variety of spectroscopy techniques. ${ }^{13-16}$ Verifying the theoretical descriptions, and understanding their modes of operation and ionic drift as well as the effects of device dimensions upon device characteristics, are significant steps in building on the usability of these devices through expanding application areas and developing a generalised model. The fact that these are nanoscale devices brings about some challenges. Conducting and analysing measurements can be more difficult, and less repeatable and reliable compared to macroscopic systems.

Studying the ionic drift is the best way to profile the switching behaviour. Electron spectroscopy techniques such as X-ray photoelectron spectroscopy (XPS) have been implemented in order to chemically address memristive characteristics. ${ }^{13,17,18}$ XPS has been predominantly used to investigate the composition of memristive devices or to carry out ex situ measurements where devices are excited prior to XPS measurements. Similar to this effort, a recent study reported in situ measurements using X-ray photoelectron emission microscopy (PEEM) to detect valence change in $\mathrm{TaO}_{x}$ devices during switching. ${ }^{13}$ Another group, sharing our motivation to better understand the switching mechanisms of memristors and ReRAMs to accelerate technological progress, has employed high-angle annular dark-field scanning transmission electron microscopy (HAADF-STEM) and twodimensional energy dispersive X-ray spectroscopy (2D EDX). ${ }^{19}$ These techniques were utilised to show the differences between a 'pristine' device (which hadn't been switched yet) and undamaged and damaged areas of a device in the low resistive state. The HAADF-STEM and EDX measurements were not in situ but 
were taken after the devices had been switched. The analysis was predominantly based on physical deformations and the changes in active layer width. These results are useful in contributing to the understanding of oxygen vacancy as well as cation movement. Other efforts have utilised imaging techniques such as scanning tunneling microscopy (STM) to investigate filament formation and how cations as well as the valence change mechanism influence switching behaviour in $\mathrm{TaO}_{x}$ memristive devices, ${ }^{20}$ in situ high-resolution transmission electron microscopy (HRTEM) to observe switching in a diffusive memristor (Pt or $\mathrm{Au}$ electrodes with $\mathrm{Ag}$ nanocrystals in $\mathrm{SiO}_{x} \mathrm{~N}_{y}$ ), ${ }^{21}$ transmission electron microscopy (TEM) to confirm different resistance states of $\mathrm{TaO}_{x}$ devices with in situ $I-V$ measurements, ${ }^{22}$ and STEM and electron energy loss spectroscopy (EELS) to observe the formation of conducting filaments in $\mathrm{SrTiO}_{3}$-based thin-film ReRAM devices. ${ }^{23}$ These results have paved the way for an in-depth understanding of different modes of memristive switching. It is important to note that, it is through these investigations of switching behaviour that it has been noticed that switching in $\mathrm{TaO}_{x}, \mathrm{HfO}_{x}$ and $\mathrm{TiO}_{x}$ devices is not solely due to the movement of oxygen vacancies but also because of the movement of cations. ${ }^{20}$ Our aim has been to carry out in situ measurements, using a variety of signals (DC, triangular, sinusoidal wave) of differing amplitudes and frequencies and observe ion movement as it is happening through XPS.

Through conducting in situ measurements, we can apply current or voltage to the device whilst observing the change in binding energy of the elements which constitute the device under test. This will shed light onto resistive switching through enabling the observation of ionic drift due to various types of $I-V$ excitation, since the nonlinearity will manifest itself through the binding energy observed through XPS. Had the active layer behaviour been linear, the binding energies of $\mathrm{Ti} 2 \mathrm{p}$ and $\mathrm{O} 1 \mathrm{~s}$ would have varied reciprocally with the excitation voltage. We report, through comparing Ti $2 \mathrm{p}$ and $\mathrm{O} 1 \mathrm{~s}$ binding energy values to that of $\mathrm{C} 1 \mathrm{~s}$, that we can chemically address the ionic movement within the memristive samples in XPS. The ultimate goal with these measurements would be to address and map each sort of switching, e.g. the way Yang et al. have outlined different kinds of switching (shunting and opening ${ }^{24}$ ), chemically through in situ XPS measurements, where we utilise the shifts in the binding energies of Ti 2p, O 1s and C 1s almost like electrical signals to see how each ion responds to the excitation signal and how their shifts in binding energy differ from one another.

XPS has advantages over other optical spectroscopy techniques since it can detect local voltage variations and give chemically specific information based on binding energy shifts. As mentioned previously, we are also able to conduct in situ measurements with XPS by applying DC or AC excitation and monitoring the resulting binding energy shifts. ${ }^{25,26}$ This setup has been recognised as being very suitable to track the ionic drift in memristive devices.

Using graphene as a top electrode has effectively enabled our measurements by allowing us to excite the memristive sample and monitor the active layer beneath graphene under excitation. The in situ $I-V$ measurements in XPS require top and bottom electrodes (to apply the excitation to and to ground, respectively), but having a metal top electrode would mean that it would not be possible to utilise XPS to get chemical data from the active layer beneath the top electrode. Utilising the transparency of graphene for XPS measurements has led to fruitful results through allowing the monitoring of the top surface of 
devices to a depth of $10 \mathrm{~nm}$, such as observing intercalation in ionic liquids on the surface. ${ }^{27}$ Graphene is a monoatomic, highly conducting and transparent material, allowing both the in situ $I-V$ measurements and the chemical XPS measurements and thus enabling chemical addressing of the nonlinearity of the device.

The two-dimensional honeycomb structure of graphene boasts many attractive electronically tunable qualities and has led to vigorous research over the past decade. Graphene exhibits high conductivity and optical transparency; it can be monoatomic in thickness or be comprised of several layers. Some applications of graphene can be summarised as follows: high speed electronics, OLED displays, smart windows, data storage, solar cells and electrochemical sensing. ${ }^{25,28}$ Due to these characteristics, graphene has been recognised as being particularly suitable to be used as transparent electrodes. Graphene boasts a better transparency to sheet resistance ratio than more traditional materials used for transparent electrodes such as indium tin oxide (ITO).$^{29}$ Graphene is becoming a widely used 2D material in ReRAMs, since it offers superior retention times and endurance compared to ReRAMs using organic polymers and it can provide devices with flexibility, whereas ITO is brittle. ${ }^{30}$ The use of graphene in applications where transparency is important is not only as electrodes but also as a modifying layer to enhance performance.$^{31}$ For example, Yang et al. reported that they layered graphene on top of ZnO for their transparent resistance random access memory (TRRAM) device and observed that the additional graphene layer offers more stable resistive switching characteristics. ${ }^{29}$ Graphene can also be used as a transparent moisture barrier, ${ }^{32}$ which would be particularly useful for devices exhibiting oxygen vacancy-based switching, since it has been demonstrated that moisture can significantly influence the performance of such devices. ${ }^{33}$

Utilising the transparency and high conductivity of graphene, we fabricated samples for in situ testing in XPS to chemically monitor the ionic movement within $\mathrm{Pt} / \mathrm{TiO}_{2} / \mathrm{TiO}_{x} /$ graphene devices. This paper briefly discusses how the devices under test have been fabricated, and the experimental setup is explained. The confirmation of the fact that these samples exhibit memristive behaviour through $I-V$ characterisation is fundamentally important to the XPS testing; as such, these devices have been characterised before the XPS measurements, and the hysteresis loops and their variation due to excitation signals with different amplitudes and frequencies are presented. The results of the in situ XPS measurements are then presented; first, the results of applying DC voltage to the device are illustrated, the resulting binding energy shifts in Ti 2p, O 1s and C 1s are shown, followed by the results of periodic triangular wave voltage excitation upon Ti 2 p and C 1 s, and finally sinusoidal voltage excitation experiment results are presented. All the XPS results show real time chemically addressed ionic movement under excitation voltage. It is new and exciting to see chemical data such as the binding energy of Ti $2 \mathrm{p}$ shifting in time due to the sinusoidal input signal, and differing from the binding energy shift of $\mathrm{C}$ 1s (graphene top electrode), thus chemically addressing hysteresis. This is a move forward for mapping the switching mechanism and hysteretic behaviour of memristive devices chemically. 


\section{Experimental}

\section{a. Fabrication of $\mathrm{Pt} / \mathrm{TiO}_{2} / \mathrm{TiO}_{x} /$ graphene XPS samples}

The device under test was fabricated similarly to nanoscale memristors and consisted of the same layers. The difference was that the devices fabricated for the purpose of XPS measurements were made larger to cater for the beam diameter of the XPS. Similarly to HP's device, ${ }^{2}$ the bottom electrode layer was platinum $(20 \mathrm{~nm}$ thick) with a chromium adhesion layer $(5 \mathrm{~nm})$. The active layer was made up of two titanium oxide layers: one layer was stoichiometric $\mathrm{TiO}_{2}$, deposited through sputtering a $\mathrm{TiO}_{2}$ target (20-30 nm thick), and the other one was $\mathrm{TiO}_{x}$, deposited by sputtering a Ti target with argon while $4-5 \%$ oxygen was simultaneously fed into the chamber (10 $\mathrm{nm}$ thick). The top electrode was a single layer of chemical vapour deposited (CVD) graphene. Graphene was grown on a copper foil, which was then etched using a $\mathrm{FeCl}_{3}$ solution. A polymer/graphene film was thus attained, which was then reverse transferred onto the active layer. ${ }^{26}$ The device structure and experimental setup is shown in Fig. 1a.

\section{b. I-V characterisation of XPS samples}

Before these macro devices could be used for measurement in XPS, they had to first be characterised to ensure that they did in fact exhibit memristive dynamics. $I-V$ characterisation was carried out using a National Instruments PXIe-1073 data acquisition unit with LabVIEW.

Fig. 2a shows clear hysteresis for two distinct sets of voltage excitation. Both voltage inputs are triangular waves with a frequency of $100 \mathrm{mHz}$. The switching behaviour can clearly be seen in the plot; the linear behaviour at both lower and higher resistance states ( $R_{\mathrm{ON}}$ and $R_{\mathrm{OFF}}$, respectively) and switching between the two states are visible for both amplitudes. Moreover, naturally the larger voltage input leads to a larger hysteresis loop. As mentioned previously, the zero crossing hysteresis loops in the first and third quadrants are evidence of passive (resistance is never negative) yet nonlinear resistance (varies with input, at least two distinct values of resistance e.g. device from Fig. $2 \mathrm{a} R_{\mathrm{ON}}=80 \Omega$ and $R_{\mathrm{OFF}}=1200$ $\Omega$ ). Fig. $2 \mathrm{~b}$ shows the effect of frequency variation and confirms that our macro memristive samples exhibit the predicted behaviour. Applying a low frequency with the same amplitude leads to larger memristance variation. Looking at

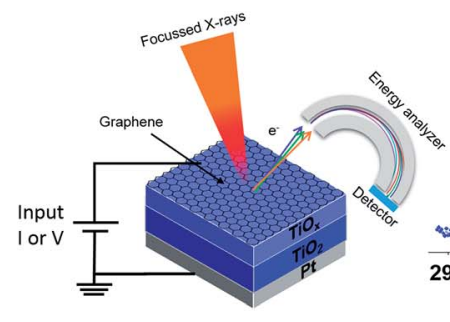

a)

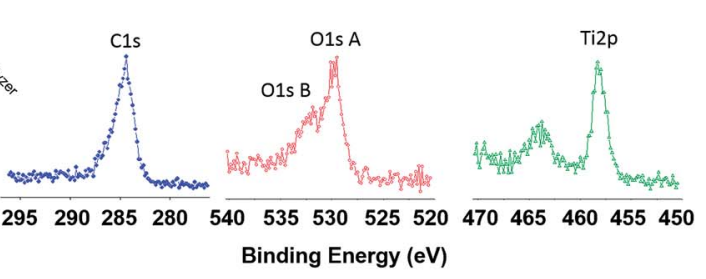

b)

Fig. 1 (a) Experimental setup of memristive device under test, with voltage or current applied in situ during XPS measurements. (b) Carbon (C 1s), oxygen (O 1s) and titanium (Ti 2p) XP spectra. 


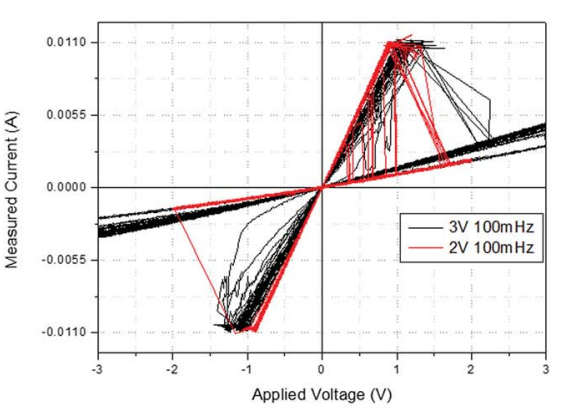

a)

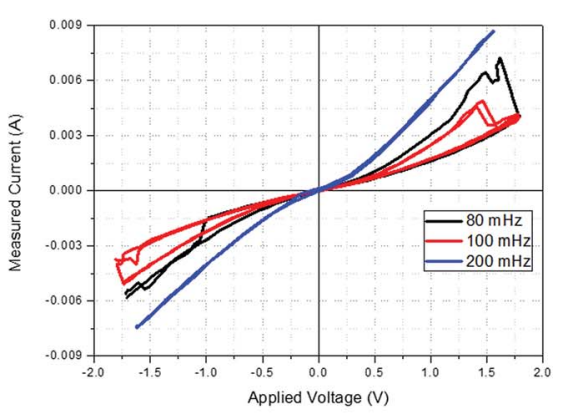

b)

Fig. 2 I-V measurement of macro memristive devices for XPS measurements. (a) The effect of amplitude variation upon hysteresis. The black and red plots show the behaviour of a device when a triangular voltage signal of $3 \mathrm{~V}$ and $2 \mathrm{~V}$ peaks, respectively, with a frequency of $100 \mathrm{mHz}$ is applied. (b) The effect of frequency variation upon hysteresis. The highest frequency, $200 \mathrm{mHz}$, for the same amplitude of triangular excitation voltage (3.4 $\mathrm{V}$ peak-to-peak) leads to the most linear behaviour (blue plot); the lowest frequency on the plot, $80 \mathrm{mHz}$, leads to the largest hysteresis loop (black plot).

a simple expression of memristance as shown in eqn (1), it is clear that the variation and value of memristance is heavily dependent on the integral of the input $(i(t)$ in eqn (1)):

$$
v(t)=M(q) i(t)=M\left(\int_{0}^{t} i(\tau) \mathrm{d} \tau\right) i(t) .
$$

Hence, if the same magnitude of excitation is applied to the device for a longer period of time (low frequency) the memristance will experience a larger variation. This leads to wider hysteresis loops. Applying inputs of higher frequencies leads to more linear behaviour, and eventually the device will act like a regular linear resistor. This trend is clearly seen in Fig. $2 \mathrm{~b}$.

\section{In situ XPS measurement results and discussion}

Having established that the samples for XPS measurements were memristive, several different experiments were conducted to track the nonlinearity and switching of these devices. DC shift measurements were performed to show the difference between the shift in carbon (graphene) and titanium (active layer) which points to nonlinearity. Moreover, AC measurements with different waveforms (triangle and sinusoidal) were applied to observe the difference in the variation of carbon and titanium. The effects of variation of frequency and amplitude upon the XP spectra of components are reported.

\section{a. DC shift in binding energies of $\mathrm{C} 1 \mathrm{~s}$, Ti 2p, O 1s A and $\mathrm{O} 1 \mathrm{~s} B$}

Binding energy shifts in $\mathrm{C} 1 \mathrm{~s}$, Ti $2 \mathrm{p}$, and both of the $\mathrm{O} 1 \mathrm{~s}$ peaks due to applied DC voltage are shown in Fig. 3. The nonlinear behaviour of the device is apparent; the slope of binding energy $v s$. applied voltage is lower for more positive applied 

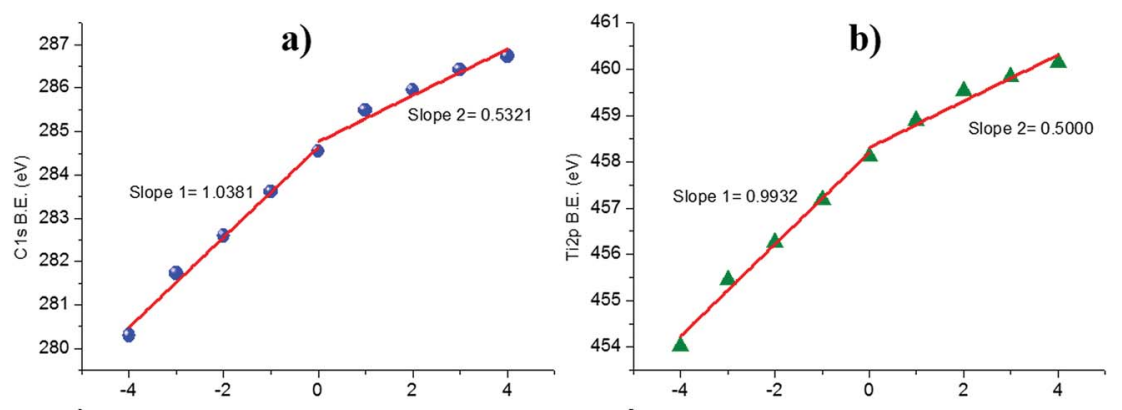

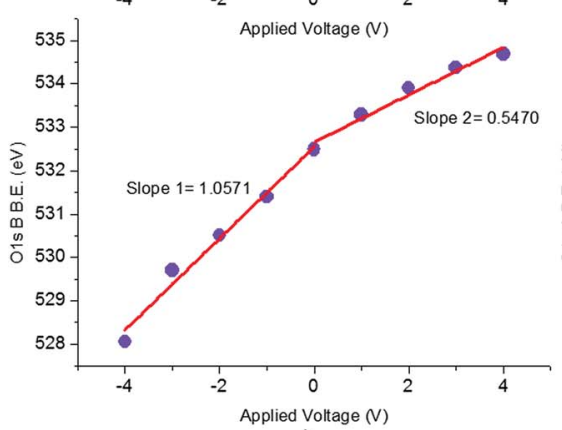

c)

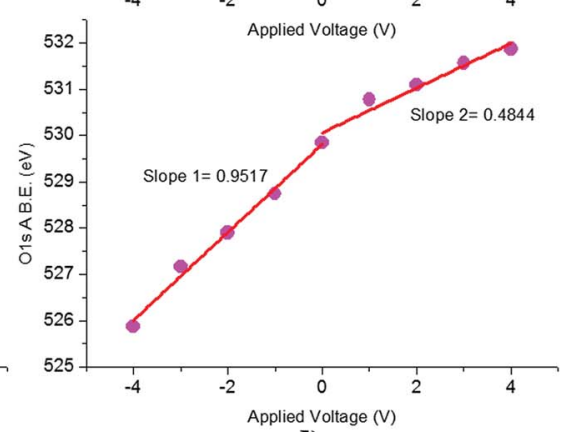

d)

Fig. 3 Binding energy shifts of C 1s (a), Ti 2p (b), and both of the $O$ 1s (O 1s A (c), O 1s B (d)) peaks due to applied DC voltage between $-4 \mathrm{~V}$ to $4 \mathrm{~V}$. Linear fits showing two distinct slopes in each plot show the correlation between $C$ 1s and $O$ 1s B, which have steeper slopes of the binding energy shift, and Ti $2 \mathrm{p}$ and $\mathrm{O} 1 \mathrm{~s} \mathrm{~A}$.

voltage. With this overall similarity between all plots in mind, the correlations between $\mathrm{C} 1 \mathrm{~s}-\mathrm{O}$ 1s B and $\mathrm{Ti} 2 \mathrm{p}-\mathrm{O}$ 1s A are evident when we compare the two slopes of each plot to one another. Hence it can be summarised that these DC shift measurements have pointed to two findings: firstly, the smaller peak of $\mathrm{O} 1 \mathrm{~s}$ (O 1s B as depicted in Fig. 1) has a similar response to that of $\mathrm{C}$ 1s from graphene. It is expected that the binding energy of graphene as the top electrode will be as close to being linear as possible, i.e. for each volt applied, the binding energy shifts $1 \mathrm{eV}$. Secondly, Ti 2p shows different variation compared to C $1 \mathrm{~s}$ as it is a component of the active layer which is responsible for the nonlinearity and switching. Looking at these results, it is possible to conclude that the behaviour of the larger peak of $\mathrm{O} 1 \mathrm{~s}$ (O 1s A as depicted in Fig. 1) points to the movement of oxygen vacancies, seeing as the slopes of variation of $\mathrm{O} 1 \mathrm{~s}$ A correlate more closely with the slopes of the Ti $2 p$ binding energy shift.

When a positive voltage is applied at the top electrode, the positively charged oxygen vacancies are drawn away from the top electrode, leaving the $\mathrm{TiO}_{2}$ layer directly underneath graphene. According to Yang et al., the device thus becomes a forward rectifier. ${ }^{24}$ Then, when a negative voltage is applied, the opposite happens: oxygen vacancies are now drawn to the negatively charged top electrode, and the device becomes a reverse rectifier. Between these two states, the $\mathrm{TiO}_{x}$ layer is sandwiched between two $\mathrm{TiO}_{2}$ layers directly adjacent to the electrodes. The in situ DC measurements demonstrate that Ti $2 \mathrm{p}$ does not simply vary with the applied voltage like $\mathrm{C}$ 1s does and that we can chemically address the oxygen 
vacancies by correlating the different shifts of the two $\mathrm{O} 1 \mathrm{~s}$ peaks to $\mathrm{C} 1 \mathrm{~s}$ and Ti 2p.

\section{b. Triangular wave voltage excitation and difference between Ti $2 \mathrm{p}$ and $\mathrm{C} 1 \mathrm{~s}$}

Expanding on the in situ XPS experiments, memristive samples were excited with periodic signals. In this section, the experiments with a triangular excitation voltage are detailed. Fig. 4a shows the line scans for Ti $2 \mathrm{p}$ and $\mathrm{C} 1 \mathrm{~s}$ whilst a triangular voltage was being applied to the device. By comparing the variation in the binding energy of $\mathrm{Ti} 2 \mathrm{p}$ to that of $\mathrm{C} 1 \mathrm{~s}$ and observing how the variation changes for different excitation signals (at different frequency and amplitudes) it is possible to inspect the effect of the oxygen vacancy drift.

Fig. $4 \mathrm{~b}$ shows the difference between the binding energy waveforms of the folded averages of three periods onto a single period of Ti $2 \mathrm{p}$ and $\mathrm{C} 1 \mathrm{~s}$. Had there been no drift within the $\mathrm{TiO}_{x}$ layer, there would be no nonlinear behaviour; thus, a constant difference in binding energies would be expected. However, as shown in Fig. 4c, not only is there a difference between the C 1s and Ti $2 p$ up slope and down slopes, but also, an increase in the C $1 \mathrm{~s}-\mathrm{Ti} 2 \mathrm{p}$ value can be clearly seen towards the end of the down slope. The difference between $\mathrm{C} 1 \mathrm{~s}$ and $\mathrm{Ti} 2 \mathrm{p}$ is clearly not constant; this points to hysteresis.

A triangular excitation voltage enables the observation of variation on the edges of the waveform. Fig. 5a shows switching in the memristive device, similar to what has been observed during $I-V$ characterization as shown in Fig. 2a. The difference between carbon and titanium is shown in Fig. 5b. It is clear that the difference between the $\mathrm{C} 1 \mathrm{~s}$ and $\mathrm{Ti} 2 \mathrm{p}$ binding energies changes due to switching. Meanwhile, the variation of carbon is obviously larger once the full triangular
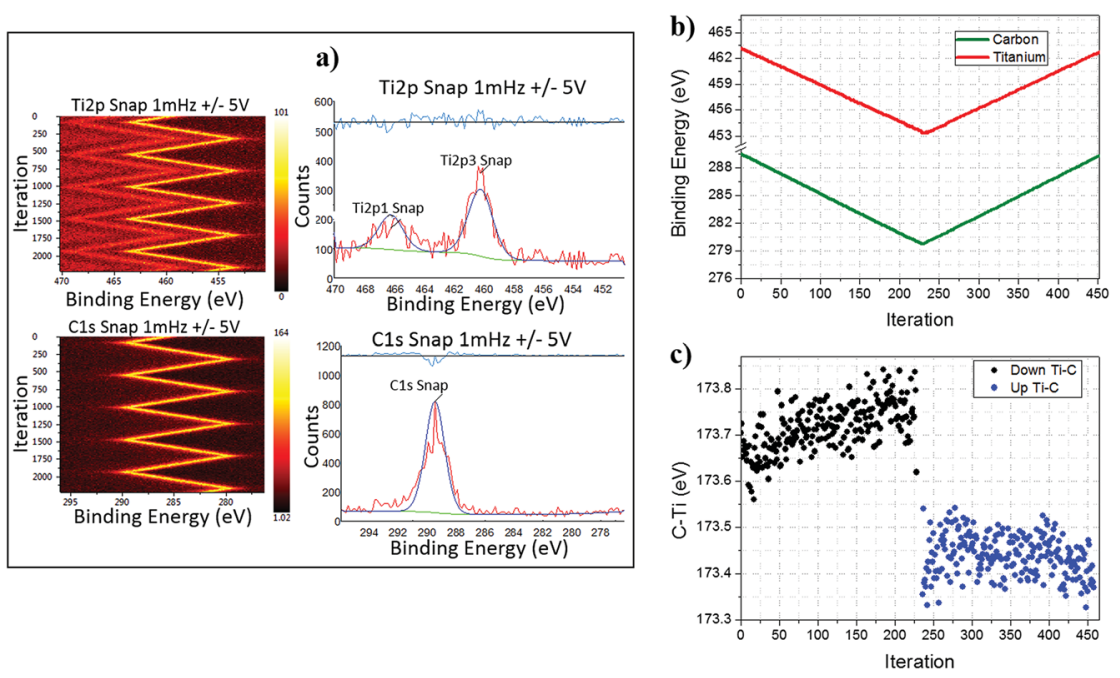

Fig. 4 (a) XPS line scan graphics for Ti $2 p$ with the doublet and C 1s with a single peak during the in situ experiment. Line scans show the triangular wave voltage applied to the device. (b) Folded averages of the binding energies of three periods of the excitation voltage ( $\pm 5 \vee 1 \mathrm{mHz}$ ) of carbon (green) and titanium (red). (c) Difference between the carbon and titanium plots, with black scatter corresponding to the difference in the down slope, and blue scatter corresponding to the difference in the up slope. 


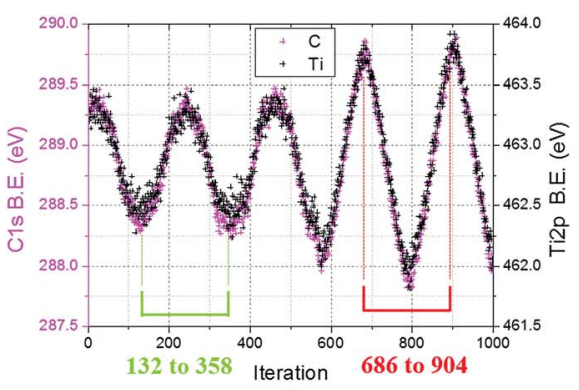

a)

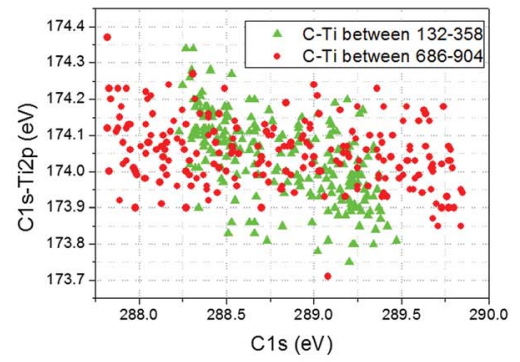

b)

Fig. 5 (a) Triangular waveform excitation of carbon and titanium showing the switching behaviour of the device, with an excitation voltage of $+/-2 \mathrm{~V}$ with $1 \mathrm{mHz}$ frequency. (b) The difference between carbon and titanium binding energies plotted against the binding energy of carbon at different sections of the transient plot. The difference shown by the red scatter plot is from the second half of the transient where the full triangular form of the waveform is realised, and hence the peak to peak variation is larger. The green scatter plot shows the first half of the transient, where variation is smaller.

form of the waveform is reached after the device has been switched on. However, prior to switching, the $\mathrm{C} 1 \mathrm{~s}-\mathrm{Ti} 2 \mathrm{p}$ is greater; the green scatter plot shows a consistent and significantly larger variation.

\section{c. Sinusoidal excitation voltage, the effect of frequency variation and chemically addressed hysteresis}

The effect of frequency upon memristive behaviour was depicted in the form of hysteresis plots in Fig. 2b. A larger frequency leads to more linear behaviour, whereas a lower frequency enhances the nonlinearity of the devices and leads to wider hysteresis loops. It was therefore expected that variation of the frequency would lead to a tangible difference in the behaviour of the Ti $2 \mathrm{p}$ XP spectra. Experiments were conducted utilising a sinusoidal excitation voltage with three distinct frequencies: $1 \mathrm{mHz}, 10 \mathrm{mHz}$ and $50 \mathrm{mHz}$. Fig. 6 illustrates the results. First and foremost, the binding energy shift of Ti $2 p$ is significantly (at least 0.2 $\mathrm{eV}$ ) lower than that of $\mathrm{C} 1 \mathrm{~s}$, which points to nonlinearity in the active layer. More importantly, the shift in the binding energy of $\mathrm{C}$ 1s remains roughly constant across the three different excitation voltage frequencies $( \pm 0.9 \mathrm{eV})$. However, the shift in Ti 2p is smallest for $50 \mathrm{mHz}( \pm 0.7 \mathrm{eV})$ and gets larger as the frequency gets lower $( \pm 0.8 \mathrm{eV})$. Intuitively from this assessment, one would expect that the difference between $\mathrm{C} 1 \mathrm{~s}$ and $\mathrm{Ti} 2 \mathrm{p}$ would be smaller for lower frequencies. However, this is not the case, as shown in Fig. $6 \mathrm{~b}$. The lowest frequency leads to the largest difference between the two components, particularly around the maxima of the sinusoid. Fig. $6 \mathrm{c}$ clarifies the reason behind this. $I-V$ measurements of memristors show that when these devices are driven by a sinusoidal voltage without DC bias, the output signal, in this case current, is a slanted sine wave, as shown by Strukov et al. ${ }^{2}$ The difference between the two periodic signals (the sinusoidal voltage and the slanted current) is the reason behind the hysteresis. If they were both perfectly sinusoidal, the resulting $I$ vs. $V$ plot would not display hysteresis, but a linear line with a slope equal to the linear and constant 


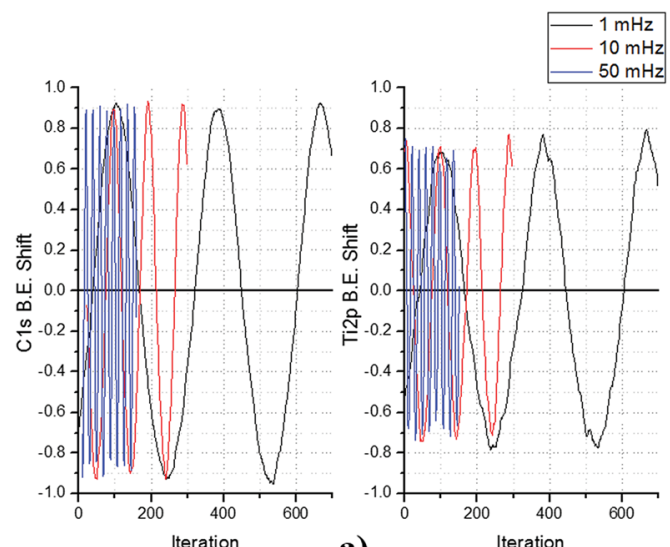

a)

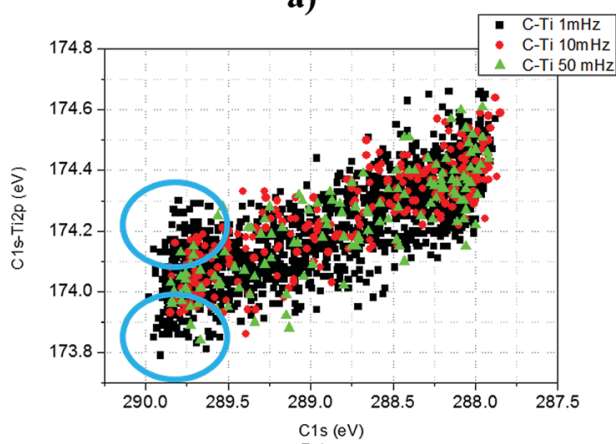

b)
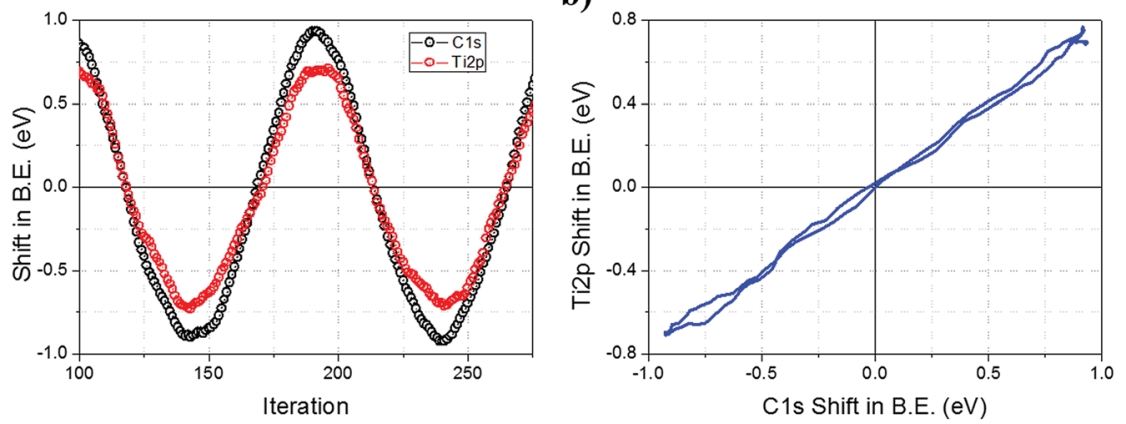

c)

Fig. 6 In situ XPS measurements with sinusoidal voltage excitation showing the effects of frequency on the XP spectra of $C 1$ s and Ti $2 p$. Panel (a) shows the shift in binding energy in the $C$ 1s and Ti $2 p$ XP spectra due to a periodic sinusoidal excitation voltage at $1 \mathrm{mHz}, 10$ $\mathrm{mHz}$ and $50 \mathrm{mHz}$ for $\pm 2 \mathrm{~V}$. Panel (b) shows the difference between $\mathrm{C} 1 \mathrm{~s}$ and Ti $2 \mathrm{p}$ plotted against the binding energy of $C 1$ s. Panel (c) shows a segment of the Ti $2 p$ and $C 1 s$ sinusoidal XP spectra as a result of the in situ measurement for the signal with a frequency of $10 \mathrm{mHz}$, and the resulting hysteresis plot when the binding energy of Ti $2 p$ is plotted against the binding energy of $C 1 \mathrm{~s}$.

resistance of the device. The comparison between the $\mathrm{C} 1 \mathrm{~s}$ and Ti $2 \mathrm{p}$ plots in Fig. $6 \mathrm{c}$ shows that $\mathrm{Ti} 2 \mathrm{p}$ is slightly slanted towards the right; it is not a perfectly symmetrical sinusoidal plot. In these results, the binding energy of $\mathrm{C} 1 \mathrm{~s}$ looks like the driving voltage in an $I-V$ measurement of a memristor and the binding energy 
of Ti $2 \mathrm{p}$ is similar to the memristive current, a slanted sine wave. The corresponding hysteresis plot showing the binding energy of $\mathrm{C} 1 \mathrm{~s}$ against $\mathrm{Ti} 2 \mathrm{p}$ is novel and demonstrative in chemically addressing hysteresis through probing ionic movement within the device. This result proves that the in situ XPS measurements performed in this endeavour can chemically track hysteresis. The advantage of working with sinusoidal signals is critical in this case; the subtle difference between the shapes of $\mathrm{C} 1 \mathrm{~s}$ and $\mathrm{Ti} 2 \mathrm{p}$ spectra are not so easily observed with triangular or square wave excitation.

\section{Conclusions}

This work focuses on chemically addressing memristive behaviour in $\mathrm{TiO}_{2} / \mathrm{TiO}_{x}$ structures with a graphene top electrode. Conducting in situ measurements with XPS enables the contents of these devices and their XP spectra to be investigated, while controllably varying current or voltage input. In other words, we are aiming to chemically characterise hysteresis with this effort, with a view of understanding the resistive switching mechanisms in such a way that will allow for a generalised model of memristive devices, paving the way for utilising them in the wide array of application fields they show promise for.

Larger samples which are more suitable for the beam size of XPS have been fabricated and characterised. The thickness of the active layer was kept nanometric to ensure that the ionic drift, responsible for memristive behaviour, could be observed in the $10 \mathrm{~nm}$ right beneath the graphene top electrode through XPS. In situ measurements were conducted. DC shift measurements were used to determine how linear the active layer is and see how Ti 2p and C 1s binding energy shifts differ and how they correlate with $\mathrm{O}$ 1s spectra. Periodic AC measurements were also taken to generate hysteresis and measure the effect in XPS. A triangular excitation voltage was applied to the device; the inconstant difference between $\mathrm{C} 1 \mathrm{~s}$ and $\mathrm{Ti} 2 \mathrm{p}$ is regarded as an indication of the nonlinearity of the active layer. Lastly, the device was driven with a sinusoidal voltage. This experiment's results clearly show that Ti $2 \mathrm{p}$ experiences considerably less binding energy shift compared to $\mathrm{C}$ 1s at higher frequencies, and the shift gets larger for lower frequencies; the difference between $\mathrm{C}$ 1s and $\mathrm{Ti} 2 \mathrm{p}$ is inconstant again, and it gets larger at lower frequencies. Looking at the plot of $\mathrm{C} 1 \mathrm{~s}$ and $\mathrm{Ti} 2 \mathrm{p}$, a slanted sinusoid reminiscent of the memristive current plots observed from $I-V$ characterisation experiments is observed.

These results are novel in that while memristive devices have been investigated with XPS to shed light onto their composition, this work reports transient in situ measurements showing chemically addressed memristivity. Shifts in binding energies of $\mathrm{C} 1 \mathrm{~s}$, Ti $2 \mathrm{p}$ and $\mathrm{O} 1 \mathrm{~s}$ have been observed and analysed to investigate and demonstrate the nonlinear behaviour of the memristive active layer. Lastly, Fig. 6c presented chemical data in a similar manner to $I-V$ measurements, showing both the slanted sinusoidal Ti $2 \mathrm{p}$ binding energy plot as a result of sinusoidal input, reminiscent of typical memristive current (as a result of sinusoidal input voltage), and a hysteresis plot of Ti $2 \mathrm{p} v s$. C $1 \mathrm{~s}$.

\section{Conflicts of interest}

There are no conflicts of interest to declare. 


\section{Acknowledgements}

This work was partially supported by the Scientific and Technological Research Council of Turkey (TUBITAK) 2218 Scheme.

\section{References}

1 L. O. Chua, Memristor - the missing circuit element, IEEE Trans. Circuit Theory, 1971, 18(5), 507-519.

2 D. B. Strukov, G. S. Snider, D. R. Stewart and S. R. Williams, The missing memristor found, Nature, 2008, 453, 80-83.

3 P. S. Georgiou, S. N. Yaliraki, E. M. Drakakis and M. Barahona, Quantitative measure of hysteresis for memristors through explicit dynamics, Proc. R. Soc. A, 2012, 468, 2210-2229.

4 Y. N. W. S. J. Joglekar, The elusive memristor: properties of basic electrical circuits, Eur. J. Phys., 2009, 30(4), 661-675.

5 B. Linares-Barranco and T. Serrano-Gotarredona, Memristance can explain spike-time-dependent-plasticity in neural synapses, Nature Precedings, 2009.

6 J. Borghetti, Z. Li, J. Straznicky, X. Li, D. A. A. Ohlberg, W. Wu, D. R. Stewart and R. S. Williams, A hybrid nanomemristor/transistor logic circuit capable of selfprogramming, Proc. Natl. Acad. Sci. U. S. A., 2009, 106(6), 1699-1703.

7 M. V. Nair, L. K. Muller and G. Indiveri, A differential memristive synapse circuit for on-line learning in neuromorphic computing systems, Nano Futures, 2017, 1(3), 035003.

8 I. Gupta, A. Serb, A. Khiat, R. Zeitler, S. Vassanelli and T. Prodromakis, Sub 100 nW volatile nano-metal-oxide memristor as synaptic-like encoder of neuronal spikes, IEEE Trans. Biomed. Circuits Syst., 2018, 12(2), 351-359.

9 G. Pedretti, V. Milo, S. Ambrogio, R. Carboni, S. Bianchi, A. Calderoni, N. Ramaswamy, A. S. Spinelli and D. Ielmini, Memristive neural network for on-line learning and tracking with brain-inspired spike timing dependent plasticity, Sci. Rep., 2017, 7, 5288.

10 S. P. Adhikari, M. P. Sah, H. Kim and L. O. Chua, Three Fingerprints of Memristor, IEEE Trans. Circuits Syst., 2013, 60(11), 3008-3021.

11 S. H. Jo, T. Chang, I. Ebong, B. B. Bhadviya, P. Mazumder and W. Lu, Nanoscale memristor device as synapse in neuromorphic systems, Nano Lett., 2010, 10(4), 1297-1301.

12 I. Valov, E. Linn, S. Tappertzhofen, S. Schmelzer, J. van den Hurk, F. Lentz and R. Waser, Nanobatteries in redox-based resistive switches require extension of memristor theory, Nat. Commun., 2013, 4, 1771.

13 A. Kindsmüller, C. Schmitz, C. Wiemann, K. Skaja, D. J. Wouters, R. Waser, C. M. Schneider and R. Dittmann, Valence change detection in memristive oxide based heterostructure cells by hard X-ray photoelectron emission spectroscopy, APL Mater., 2018, 6(4), 046106.

14 F. Miao, J. P. Strachan, J. J. Yang, M. Zhang, I. Goldfarb, A. C. Torrezan, P. Eschbach, R. D. Kelley, G. Medeiros-Ribeiro and R. S. Williams, Anatomy of a Nanoscale Conduction Channel Reveals the Mechanism of a HighPerformance Memristor, Adv. Mater., 2011, 23(47), 5633-5640.

15 S. Kumar, C. E. Graves, J. P. Strachan, E. M. Grafals, A. L. D. Kilcoyne, T. Tyliszczak, J. N. Weker, Y. Nishi and W. R. Stanley, Direct Observation of 
Localized Radial Oxygen Migration in Functioning Tantalum Oxide Memristors, Adv. Mater., 2016, 28(14), 2772-2776.

16 R. Waser and M. Aono, Nanoionics-based resistive switching memories, Nat. Mater., 2007, 6, 833-840.

17 P. Bousoulasa, I. Giannopoulos, P. Asenov, I. Karageorgiou and D. Tsoukalas, Investigating the origins of high multilevel resistive switching in forming free $\mathrm{Ti} / \mathrm{TiO}_{2-x}$-based memory devices through experiments and simulations, $J$. Appl. Phys., 2017, 121(9), 094501.

18 C. Li, Y. Vaynzof, G. Lakhwani, G. J. Beirne, J. Wang and N. C. Greenham, Observation of oxygen vacancy migration in memory devices based on $\mathrm{ZnO}$ nanoparticles, J. Appl. Phys., 2017, 121, 144503.

19 D. Carta, I. Salaoru, A. Khiat, A. Regoutz, C. Mitterbauer, N. M. Harrison and T. Prodromakis, Investigation of the Switching Mechanism in TiO2-Based RRAM: A Two-Dimensional EDX Approach, J. Am. Chem. Soc., 2016, 8(30), 19605-19611.

20 A. Wedig, M. Luebben, D.-Y. Cho, M. Moors, K. Skaja, V. Rana, T. Hasegawa, K. K. Adepalli, B. Yildiz, R. Waser and I. Valov, Nanoscale cation motion in TaOx, HfOx and TiOx memristive systems, Nat. Nanotechnol., 2015, 11, 67-75.

21 Z. Wang, S. Joshi, S. E. Savel'ev, H. Jiang, R. Midya, P. Lin, M. Hu, N. Ge, J. P. Strachan, Z. Li, Q. Wu, M. Barnell, G.-L. Li, H. L. Xin, R. S. Williams, Q. Xia and J. J. Yang, Memristors with diffusive dynamics as synaptic emulators for neuromorphic computing, Nat. Mater., 2016, 16, 101-110.

22 G.-S. Park, Y. B. Kim, S. Y. Park, X. S. Li, S. Heo, M.-J. Lee, M. Chang, J. H. Kwon, M. Kim, U.-I. Chung, R. Dittmann, R. Waser and K. Kim, In situ observation of filamentary conducting channels in an asymmetric $\mathrm{Ta}_{2} \mathrm{O}_{5-x} /$ $\mathrm{TaO}_{2-x}$ bilayer structure, Nat. Commun., 2013, 4, 2382.

23 H. Du, C.-L. Jia, A. Koehl, J. Barthel, R. Dittmann, R. Waser and J. Mayer, Nanosized Conducting Filaments Formed by Atomic-Scale Defects in RedoxBased Resistive Switching Memories, J. Am. Chem. Soc., 2017, 29(7), 3164-3173.

24 J. Yang, J. Borghetti, D. Murphy, D. Stewart and S. R. Williams, A Family of Electronically Reconfigurable Nanodevices, Adv. Mater., 2009, 21(37), 37543758.

25 M. Copuroglu, P. Aydogan, E. O. Polat, C. Kocabas and S. Suzer, Gate-Tunable Photoemission from Graphene Transistors, Nano Lett., 2014, 14(5), 2837-2842.

26 P. Aydogan Gokturk, N. Kakenov, C. Kocabas and S. Suzer, Raman and X-Ray photoelectron spectroscopic studies of graphene devices for identification of doping, Appl. Surf. Sci., 2017, 425, 1130-1137.

27 O. Salihoglu, H. B. Uzlu, O. Yakar, S. Aas, O. Balci, N. Kakenov, S. Balci, S. Olcum, S. Suzer and C. Kocabas, Graphene-Based Adaptive Thermal Camouflage, Nano Lett., 2018, 18(7), 4541-4548.

28 E. P. Randviir, D. A. C. Brownson and C. E. Banks, A decade of graphene research: production, applications and outlook, Mater. Today, 2014, 17(9), 426-432.

29 P. K. Yang, W. Y. Chang, P. Y. Teng, S. F. Jeng, S. J. Lin, P. W. Chiu and J. H. He, Fully Transparent Resistive Memory Employing Graphene Electrodes for Eliminating Undesired Surface Effects, Proc. IEEE, 2013, 101(7), 1732-1739.

30 F. Hui, E. Grustan-Gutierrez, S. Long, Q. Liu, A. K. Ott, A. C. Ferrari and L. Mario, Graphene and Related Materials for Resistive Random Access Memories, Adv. Electron. Mater., 2017, 3(8), 1600195. 
31 Y. Xu and J. Liu, Graphene as Transparent Electrodes: Fabrication and New Emerging Applications, Small, 2016, 12(11), 1400-1419.

32 S. Won, D. Van Lam, J. Y. Lee, H.-J. Jung, M. Hur, K.-S. Kim, H.-J. Lee and J.-H. Kim, Graphene-based stretchable and transparent moisture barrier, Nanotechnology, 2018, 29(12), 125705.

33 F. Messerschmitt, M. Kubicek and J. L. Rupp, How Does Moisture Affect the Physical Property of Memristance for Anionic-Electronic Resistive Switching Memories?, Adv. Funct. Mater., 2015, 25(32), 5117-5125. 